Clinical Medicine \& Research

Volume 1, Number 3: 227-232

\title{
Thymoma and Multiple Malignancies: A Case of Five Synchronous Neoplasms and Literature Review
}

\author{
James S. Welsh, MS, MD, Department of Human Oncology, University of Wisconsin School of Medicine, Madison, Wisconsin \\ Sarah A. Thurman, MD, Department of Radiation Oncology, Beth Israel-Deaconess Medical Center, Boston, Massachusetts \\ Steven P. Howard, MD, PhD, Department of Human Oncology, University of Wisconsin School of Medicine, Madison, Wisconsin
}

\begin{abstract}
The presence of five discrete synchronous or metachronous primary neoplasms in a single patient is an extremely rare event. This is a report of a patient with a malignant (invasive) thymoma and four other independent primary neoplasms including: gliosarcoma, papillary thyroid cancer, meningioma and metastatic adenocarcinoma of the colon, found synchronously at autopsy. Thymoma patients appear to have an inherent predisposition towards developing additional neoplasms. Other than the thymoma, the presented patient had no obvious risk factors for neoplasia. This case provides evidence for an unusual syndrome of thymoma and multiple primary neoplasms. Further research is required to elucidate the mechanism of this association. Meanwhile, heightened awareness of this association may allow earlier detection and treatment of additional cancers in patients with a history of thymoma.
\end{abstract}

\section{INTRODUCTION}

The development of five primary tumors in a single patient is extremely uncommon. Finding the five neoplasms synchronously is an extraordinarily rare event. Although cases of five or more synchronous primary tumors have been reported, these cases typically include multiple discrete tumors within the same or contralateral-paired organ. A patient who died of gliosarcoma and was found to synchronously have a total of five independent primary neoplasms including gliosarcoma, papillary thyroid cancer, meningioma, adenocarcinoma of the colon with hepatic metastases, and thymoma, is presented. Gliosarcoma itself is a highly aggressive, rarely encountered tumor of the central nervous system. Synchronous association of this unusual neoplasm with four other distinct neoplasms is exceptional, and to our knowledge, not previously reported. Such a peculiar array of multiple primary tumors appearing synchronously seems unlikely to be merely coincidental, but is suggestive of carcinogen exposure, or perhaps a sort of a "cancer syndrome." This particular patient was without a history of carcinogen exposure, a prior personal history of cancer, or a familial predisposition. The presence of the thymoma is intriguing. A report from the Johns Hopkins Hospital ${ }^{1}$ found additional neoplasms in $31 \%$ of thymoma patients at some time during their follow-up. Other large series ${ }^{2-8}$ have similarly observed thymomas to be associated with additional non-thymic neoplasms with incidence rates ranging from $8 \%$ to $21 \%$. Other than the thymoma, the patient had no obvious risk factors for neoplasia, providing further evidence for this unusual syndrome of thymoma and multiple primary malignancies.

RECEIVED: FEBRUARY 2, 2003

REPRINT REQUESTS:

James S. Welsh, MD, PhD

Department of Human Oncology

University of Wisconsin-Madison

600 Highland Avenue

$\mathrm{K} 4 / \mathrm{B} 100$

Madison, WI 53792

Telephone: 608-263-8500

Fax: 608-263-9167

Email:welsh@humonc.wisc.edu
REVISED AND ACCEPTED: MARCH 6, 2003

KEYWORDS:

Multiple primary neoplasms; Synchronous neoplasms; Thymoma 


\section{CASE PRESENTATION}

A female, 85 years of age, in good general health, presented to a local emergency department with an acute onset of left hemi-paresis. The patient was clinically diagnosed with an acute right cerebral hemispheric stroke. After being stabilized, the patient was transferred to Johns Hopkins Hospital for further management. Brain magnetic resonance imaging (MRI) identified a $3 \times 2-\mathrm{cm}$ right parietal lesion and a 1.5$\mathrm{cm}$ right frontal lesion, both causing mass effect. Although the findings were compatible with hemorrhagic metastases, the differential diagnosis also included separate primary brain tumors. Radiographic studies including chest x-ray, bilateral mammogram, abdomen and pelvis were unremarkable. The carcinoembryonic antigen (CEA) was elevated to a level of $23 \mathrm{ng} / \mathrm{ml}$. Other labs including thyroid function tests were within normal limits except for a slightly elevated alkaline phosphatase. Both the patient and her family decided to not pursue further work-up at that time. The patient was in stable condition and discharged home.

The patient's past medical history was notable for hypertension, mild dementia, depression, anemia, glaucoma and congestive heart failure. The patient had an aortic graft in the past, as well as a hysterectomy. There was no history of prior malignancy or family history of cancer. There was no record of carcinogen exposure.

The patient was readmitted two months later because of progressive left-sided hemi-paresis and mental status changes. Repeat brain MRI showed a large right hemispheric cystic tumor measuring $5.4 \times 4 \times 3 \mathrm{~cm}$ with massive peritumoral edema and mass effect, causing midline shift and subfalcine herniation. Given steady decline in performance status, the patient's family chose to only pursue supportive treatment. The patient was placed in hospice and died two months later.

Autopsy revealed 5 discrete primary tumors. The cause of death was attributed to a primary brain tumor, which was diagnosed as gliosarcoma by light microscopy and immunohistochemical staining. The patient was also noted to have a 2-cm, high-grade, poorly differentiated adenocarcinoma of the cecum invading through the muscularis and into the surrounding fat. Multiple liver metastases were found and attributed to the cecal primary. The third primary was a follicular variant of papillary thyroid carcinoma of the inferior right thyroid lobe measuring $1 \times 1 \times 1 \mathrm{~cm}$. The fourth primary tumor was a right frontal lobe meningioma measuring $1.5 \times 1 \times 1 \mathrm{~cm}$. The fifth and final primary was a malignant (invasive) thymoma, measuring $3 \times 2 \times 2 \mathrm{~cm}$ and extending into the anterior pericardial fat.

\section{DISCUSSION}

\section{Multiple Neoplasms}

Occult thyroid carcinomas found at autopsy are not rare. Autopsy series have revealed incidental thyroid cancers in about $10 \%$ of cases. ${ }^{9,10}$ Incidental meningiomas are also found in autopsy series at slightly over $1 \%{ }^{1}$ The probability of finding both in the same patient is simply the product of these two prevalences $(0.1 \%)$. The likelihood of also simultaneously discovering a gliosarcoma, metastatic colorectal adenocarcinoma and invasive thymoma in the same patient is exceedingly small. A review of 140,000 cancer patients identified only one patient $(0.0007 \%)$ with 5 discrete primary malignancies. ${ }^{12}$ Rohde and Jakse ${ }^{13}$ reviewed the literature on multiple primary malignant neoplasms and found 16 patients with 5 or more distinct primary tumors. Excluding cases in which separate tumors were reported in the same organ or in the contralateral-paired organ, only 10 patients with 5 or more discrete primary neoplasms, including their own case were identified. However, in 7 of these cases, the neoplasms developed metachronously, in contrast to the synchronous appearance in our case. The extreme rarity of the current case is suggestive of an underlying mechanism, rather than a mere chance association.

\section{Etiology of Multiple Neoplasms}

Gliosarcoma is considered a rare variant of glioblastoma multiforme, constituting $1.8 \%$ to $2.4 \%$ of glioblastoma cases in large series. ${ }^{14,15}$ It has been reported in association with prior exposure to ionizing radiation. ${ }^{16,17}$ Meningiomas ${ }^{18-20}$ and thyroid cancers ${ }^{21-23}$ likewise have been linked with radiation exposure. In addition to radiation, chemotherapy is well-known to be associated with secondary malignancies in cancer patients. ${ }^{24-27}$ The presented patient never received any radiotherapy or chemotherapy for symptomatic gliosarcoma or any of the tumors; thus, iatrogenic carcinogenesis can not explain the remarkable constellation of neoplasms.

Additional cancers are known to develop at a higher rate in people with a prior personal history of cancer. Patients with primary head and neck cancers occasionally develop additional tumors, presumably due to a history of heavy smoking and/or alcohol consumption, resulting in a "field cancerization" effect, predisposing the entire upper aerodigestive tract to additional cancers. ${ }^{28,29}$ The additional neoplasms in these cases are typically squamous cell carcinomas restricted to the upper aerodigestive system. The elevated risk of second cancers in patients with a personal history of cancer cannot always be explained by exogenous carcinogen exposure. Developmental and genetic factors play a role in some patients. Patients with ovarian and breast cancers are at higher risk for development of cancer in the contralateral paired organ, perhaps because of an endogenous hormonal imbalance and/or a genetic predisposition. While patients with Hodgkin's disease occasionally develop second malignancies after cytotoxic therapies, a baseline analysis revealed abnormalities in sister chromatid exchange in some patients, which was independently predictive of the development of a second cancer. ${ }^{30}$ These abnormalities were identified before any treatment was administered. Treatment itself was not found to be an independent risk for second cancers, supporting the notion of an inherent predisposition to neoplasia in patients with Hodgkin's disease. Well-studied inherited mutations such as p53 (Li-Fraumeni syndrome), ${ }^{31}$ mismatch-repair genes in Lynch syndromes ${ }^{32}$ and $\mathrm{Rb}$ protein (p105-RB), in retinoblastoma, ${ }^{33}$ predispose individuals 
and their families to a variety of multiple malignancies. This patient did not have a history of smoking, alcohol abuse, carcinogen exposure, or a personal or family history of cancer. However, the patient did have a thymoma.

\section{Thymoma and Additional Neoplasms}

Several series have confirmed an increased incidence of thymoma and additional neoplasms (table 1), with prevalence rates as high as $31 \% .^{1}$ The discovery of multiple synchronous neoplasms along with thymoma has also been reported in the veterinary literature. In a study of 23 dogs with thymoma, additional neoplasms were concurrently found among 5 dogs (22\%), paralleling the human observations. ${ }^{34}$ In people, thymoma has been found to be associated with additional malignant neoplasms of various sorts, most notably colorectal adenocarcinoma and thyroid cancer. ${ }^{1,8} \mathrm{At}$ the Mayo Clinic, Souadjian et al. ${ }^{6}$ found that $21 \%$ of 146 thymoma patients developed second malignancies. This was compared and contrasted with an $8 \%$ second malignancy rate among 177 patients with parathyroid tumors. Their review of the literature ${ }^{7}$ revealed a reported second malignancy incidence of $17 \%$ among patients surviving beyond 5 years after diagnosis of thymoma. The true incidence of additional malignancies was higher, as cases with simultaneously diagnosed lymphoma or leukemia were excluded to avoid potential confusion between thymoma and secondary lymphocytic infiltration of the thymus. Interestingly, this population (thymoma plus leukemia or lymphoma) accounted for a high proportion (10\%) of the 588 evaluable patients, a finding observed by others. ${ }^{35,36}$ In Taiwan, Pan et al. ${ }^{5}$ also found an increased risk of second malignancies among thymoma patients. In their study, the second malignancy incidence rate among thymoma patients was significantly higher compared to a matched cohort of patients with nasopharyngeal carcinoma ( $8 \%$ vs. $2 \%)$. The largest single series reporting thymoma and additional neoplasms was conducted by Lewis et al. ${ }^{4}$ who reviewed the Mayo Clinic experience and revealed a second malignancy rate of $17 \%$ (48 of 283), corroborating the prior work of Souadjian et al. ${ }^{6,7}$ A recent review ${ }^{5}$ confirmed a $17 \%$ (152 of 909) incidence of second malignancies in patients with thymoma with colorectal carcinomas representing the most common site. The current review resulted in an identical $17 \%$ figure (table 1).

\section{Treatment-Induced Malignancies}

As the early reports did not specify treatment for the thymomas, the possibility of treatment-induced neoplasia remained. Welsh et al. ${ }^{1}$ showed that the high incidence of additional neoplasms in thymoma patients cannot be attributed to adjuvant radiotherapy or chemotherapy and appears to be an intrinsic association. Of 142 thymoma patients, 46 received radiation or chemotherapy. Among these 46 patients, 16 (35\%) developed second neoplasms. This did not differ significantly from the prevalence of second neoplasms in those who did not receive radiation or chemotherapy (25\%), or the thymoma population as a whole (28\%). Conversely, of the 38 patients with additional neoplasms, only 16 received radiation or chemotherapy, while 22 did not. Among patients who received radiotherapy and developed additional neoplasms, the second tumors usually developed outside the radiation fields, a finding confirmed by others, ${ }^{5}$ which strongly argues against a link to the radiotherapy. Finally, as in the present case, the other tumors were often diagnosed shortly after, synchronously, or even before the thymoma, again not consistent with treatment-induced neoplasia. Thus, radiation or chemotherapy apparently does not account for the high rate of additional neoplasms in thymoma patients.

Because of the integral role of the thymus in immunity, one may speculate that surgical removal of the thymus may be predisposing thymoma patients to additional malignancies. However, Bulkley et al. ${ }^{37}$ observed no increase in malignancies among patients who underwent thymic resection for myasthenia gravis, except when the myasthenia gravis was accompanied by thymoma. ${ }^{8}$ Similarly, Masaoka et al. ${ }^{38}$ analyzed 390 patients with myasthenia gravis who had undergone thymic resections and found no increase in the extrathymic malignancy rate among those without an associated thymoma. In contrast, patients with thymoma and myasthenia gravis who underwent thymic resection had an observed-to-expected malignancy ratio of $3: 42$. Pan et al. ${ }^{5}$ also found a higher rate of additional tumors in patients undergoing thymectomy for thymoma ( $8 \%$ ) compared to patients who had thymectomy for myasthenia gravis alone $(2 \%)$. Thus surgical resection of the thymus for myasthenia gravis apparently does not increase the chances of developing cancer.

Table 1. Series involving more than 50 patients with thymoma and reporting data on prevalence of additional malignancies. ${ }^{*}$

\begin{tabular}{|c|c|c|c|c|}
\hline Study & Number of patients & Institution & Prevalence & Percent \\
\hline Lewis et $\mathrm{al}^{4}$ & 283 & Mayo Clinic & $48 / 283$ & $17 \%$ \\
\hline Pan et $\mathrm{al}^{5}$ & 192 & leterans General Hospital, Taipei, Taiwan & $15 / 192$ & $8 \%$ \\
\hline Welsh et $\mathrm{al}^{1}$ & 142 & Johns Hopkins Hospital & $44 / 142$ & $31 \%$ \\
\hline Gray and Gutowski ${ }^{3}$ & 54 & New York Hospital & $5 / 54$ & $9 \%$ \\
\hline Couture and Mountain ${ }^{2}$ & Univ & hiversity of Texas, M.D. Anderson Cancer Center & $11 / 52$ & $21 \%$ \\
\hline Total & 723 & & $123 / 723$ & $17 \%$ \\
\hline
\end{tabular}

${ }^{*}$ Other series ${ }^{6,8}$ from the literature were excluded if their patent population was included in the larger series from the same institution. 


\section{Associated Paraneoplastic Disorders}

The paraneoplastic conditions typically linked with thymoma such as myasthenia gravis, red cell aplasia and hypogammaglobulinemia represent immunological disorders and thus could conceivably be related to the higher rates of cancer either intrinsically, or because of immunosuppressive therapy for the autoimmune disorder. The presented patient did not have any obvious thymoma-associated paraneoplastic immunological phenomenona. Wilkins et al. ${ }^{8}$ and others, ${ }^{1}$ found that the presence or absence of paraneoplastic immunological disorders did not appear to influence the development of further neoplasms. Of 67 patients with immunological disorders, only $24 \%$ developed additional tumors-no greater than in the total thymoma population studied (38 of 135 [28\%]) or in the thymoma patients without associated disorders (21 of 68 [31\%]). Thus, the presence of (or treatment for) such thymoma-associated conditions cannot entirely explain the high malignancy rate.

\section{Multiple Malignancies Associated with Thymoma}

The finding of multiple malignancies in human patients $1,5,39$ and in $\operatorname{dogs}{ }^{34}$ with thymoma supports the concept of an inherent, rather than coincidental link between thymoma and cancer. This strong oncogenic tendency among patients with thymoma is illustrated by a review by Freidman et al. ${ }^{39}$ in which a third discrete malignancy developed in 33\% of patients with a history of thymoma and a hematologic neoplasm. Very similar findings were reported from Johns Hopkins, ${ }^{1}$ where 58 additional neoplasms were found in 44 thymoma patients. A total of 14 patients (31\%) were found to have 3 or more discrete primary tumors either synchronously, or metachronously during follow-up. Our present case with 5 synchronous distinct primary neoplasms provides an extreme example of this susceptibility.

\section{Pathogenesis}

A precise pathogenic mechanism linking thymoma to an increased incidence of cancer remains elusive. Studies have demonstrated histologic differences in thymic tissue from cancer patients compared to normal controls. ${ }^{40}$ Although this may implicate thymic function in some way; it is unclear if this is a cause or effect relationship. The authors postulated that development of thymoma implies a defect in thymic epithelium that hinders T-cell development, leading to immune defects and a higher incidence of cancer. Some thymoma patients reportedly have peripheral T-cell lymphocytosis, which may reflect a perturbation of systemic immunoregulation that accompanies thymic neoplasia. ${ }^{41}$ Such immune dysfunction could theoretically lead to a breakdown in immune surveillance allowing uncontrolled proliferation of neoplasms, which would otherwise be kept in check. Skinnider et al. ${ }^{36}$ have suggested that decreased natural killer cell function resulting from increased suppressor T-cell activity could be causally linked to the increased incidence of cancer in patients with thymoma. Freidman et al. ${ }^{39}$ proposed that the thymoma epithelium may continuously stimulate T-lymphocytes, which in turn predisposes to neoplasia. While this may account for cases of hematologic malignancies, it does not adequately explain the various solid tumors encountered, nor does it explain the tumors observed before or synchronously with the thymoma.

Although cytogenetic abnormalities have been reported in thymoma, ${ }^{44,45}$ presently no molecular or cytogenetic mechanisms adequately explain the tendency of thymoma patients to acquire additional neoplasms. Interestingly, patients with Hodgkin's disease also have a high incidence of second malignancies. 25,27 While it is assumed that this high incidence of additional malignancies is due to radiation or chemotherapy, baseline cytogenetic studies of patients with Hodgkin's disease before treatment have identified abnormalities in sister chromatid exchange as an independent risk factor for second primary cancers. ${ }^{30}$ Occasionally, Hodgkin's disease occurs synchronously with other neoplasms, suggesting that like thymoma, some of these patients have an intrinsic predisposition towards additional cancers. ${ }^{42}$ Curiously, as this case, a patient with Hodgkin's disease as one of 5 distinct malignant neoplasms has been reported, ${ }^{13}$ as has a patient with gliosarcoma developing after Hodgkin's disease. 43

\section{CONCLUSION}

The presence of 5 or more distinct metachronous primary neoplasms in a single patient is a very rare event. The detection of 5 or more primary neoplasms simultaneously in a single patient is extraordinary. There is now convincing evidence of a syndrome associating thymoma with additional cancers. Most cases of second malignancies appear metachronously, with an overall prevalence of $17 \%$. Adenocarcinomas of the gastrointestinal tract appear most frequently but a wide array of different histologies has been observed. The precise pathogenic mechanism remains unclear. We suspect that the presented patient's thymoma was related to the multiple neoplasms by either inducing a proclivity towards neoplasia or serving as a marker for an inherent propensity for neoplasia. Although medical texts routinely describe the classic associations of thymoma with myasthenia gravis, pure red cell aplasia, and hypogammaglobulinemia, there is also an important association between thymoma and malignant neoplasms. The existence of this syndrome is illustrated by this case and is supported by a review of the medical and veterinary literature. For patients with a past history of thymoma, awareness of this association should allow earlier detection and treatment of any future cancers through proper surveillance.

\section{ACKNOWLEDGMENTS}

The authors gratefully acknowledge and appreciate the assistance of Anne Kammer of the Johns Hopkins Tumor Registry. 


\section{REFERENCES}

1. Welsh JS, Wilkins KB, Green R, Bulkley G, Askin F, DienerWest M, Howard SP. Association between thymoma and second neoplasms. JAMA 2000;283:1142-1143.

2. Couture MM, Mountain CF. Thymoma. Semin Surg Oncol 1990;6:110-114.

3. Gray GF, Gutowski WT 3rd. Thymoma. A clinicopathologic study of 54 cases. Am J Surg Pathol 1979;3:235-249.

4. Lewis JE. Wick MR. Scheithauer BW. Bernatz PE. Taylor WF. Thymoma. A clinicopathologic review. Cancer 1987;60: 2727-2743.

5. Pan CC, Chen PC, Wang LS, Chi KH, Chiang H. Thymoma is associated with an increased risk of second malignancy. Cancer 2001;92:2406-2411.

6. Souadjian JV, Silverstein MN, Titus JL. Thymoma and cancer. Cancer 1968;22:1221-1225.

7. Souadjian JV, Enriquez P, Silverstein MN, Pepin JM. The spectrum of diseases associated with thymoma. Coincidence or syndrome? Arch Intern Med 1974;134:374-379.

8. Wilkins KB, Sheikh E, Green R, Patel M, George S, Takano M, Diener-West M, Welsh J, Howard S, Askin F, Bulkley GB. Clinical and pathologic predictors of survival in patients with thymoma. Ann Surg 1999;230:562-572.

9. Bondeson L, Ljungberg O. Occult thyroid carcinoma at autopsy in Malmo, Sweden. Cancer 1981;47:319-323.

10. Chong PY Thyroid carcinomas in Singapore autopsies. Pathology 1994;26:20-22.

11. Annegers JF, Schoenberg BS, Okazaki H, Kurland LT. Epidemiologic study of primary intracranial neoplasms. Arch Neurol 1981;38:217-219.

12. Mersheimer WL, Ringel A, Eisenberg H.: Some characteristics of multiple primary cancers. Ann NY Acad Sci 1964;114: 896-921.

13. Rohde D, Jakse G. Involvement of the urogenital tract in patients with five or more separate malignant neoplasms. Case and review. Eur Urol 1998;34:512-517.

14. Galanis E, Buckner JC, Dinapoli RP, Scheithauer BW, Jenkins RB, Wang CH, O'Fallon JR, Farr G Jr. Clinical outcome of gliosarcoma compared with glioblastoma multiforme: North Central Cancer Treatment Group results. J Neurosurg 1998; 89:425-430.

15. Meis JM, Martz KL, Nelson JS. Mixed glioblastoma multiforme and sarcoma. A clinicopathologic study of 26 radiation therapy oncology group cases. Cancer 1991;67:2342-2349.

16. Kaschten B, Flandroy P, Reznik M, Hainaut H, Stevenaert. A Radiation-induced gliosarcoma. Case report and review of the literature. J Neurosurg 1995;83:154-162.

17. Lach M, Wallace CJ, Krcek J, Curry B. Radiation-associated gliosarcoma. Can Assoc Radiol J 1996;47:209-212.

18. Hu J, Little J, Xu T, Zhao X, Guo L, Jia X, Huang G, Bi D, Liu R. Risk factors for meningioma in adults: a case-control study in northeast China. J Int J Cancer 1999;83:299-304.

19. Sadetzki S, Modan B, Chetrit A, Freedman L. An iatrogenic epidemic of benign meningioma. Am J Epidemiol 2000;151:266-272.

20. Shintani T, Hayakawa N, Hoshi M, Sumida M, Kurisu K, Oki S, Kodama Y, Kajikawa H, Inai K, Kamada N. High incidence of meningioma among Hiroshima atomic bomb survivors. Radiat Res 1999;40:49-57.

21. Greenspan FS. Radiation exposure and thyroid cancer. JAMA 1977;2379:2089-2091.

22. Schlumberger M, Cailleux AF, Suarez HG, de Vathaire F. Irradiation and second cancers. The thyroid as a case in point. C R Acad Sci III 1999;322:205-213.

23. Pacini F, Vorontsova T, Molinaro E, Shavrova E, Agate L, Kuchinskaya E, Elisei R, Demidchik EP, Pinchera A. Thyroid consequences of the Chernobyl nuclear accident. Acta Paediatr Suppl 1999;88:23-27.
24. Kushner BH, Heller G, Cheung NK, Wollner N, Kramer K, Bajorin D, Polyak T, Meyers PA. High risk of leukemia after short-term dose-intensive chemotherapy in young patients with solid tumors. J Clin Oncol 1998;16:3016-3020.

25. Swerdlow AJ, Barber JA, Hudson GV, Cunningham D, Gupta RK, Hancock BW, HorwichA, Lister TA, Linch DC. Risk of second malignancy after Hodgkin's disease in a collaborative British cohort: the relation to age at treatment. J Clin Oncol 2000;18:498-509.

26. Tucker MA, Murray N, Shaw EG, Ettinger DS, Mabry M, Huber MH, Feld R, Shepherd FA, Johnson DH, Grant SC, Aisner J, Johnson BE. Second primary cancers related to smoking and treatment of small-cell lung cancer. Lung Cancer Working Cadre. J Natl Cancer Inst 1997;89:17821788 .

27. van Leeuwen FE, Klokman WJ, Veer MB, Hagenbeek A, Krol AD, Vetter UA, Schaapveld M, van Heerde P, Burgers JM, Somers R, Aleman BM. Long-term risk of second malignancy in survivors of Hodgkin's disease treated during adolescence or young adulthood. J Clin Oncol 2000;18:487-497.

28. Kiriu H, Yokozaki H, Yasui W, Ito K, Tahara E. Microsatellite instability associated with primary head and neck cancers and secondary esophageal cancers. Jpn J Clin Oncol 1998;28:733-739.

29. Lydiatt WM, Anderson PE, Bazzana T, Casale M, Hughes CJ, Huvos AG, Lydiatt DD, Schantz SP. Molecular support for field cancerization in the head and neck. Cancer 1998;82:1376-1380.

30. Strom SS, Gu Y, Sigurdson AJ, Bailey NM, Amos CI, Spitz MR, Rodriguez MA, Liang JC. Chromosome breaks and sister chromatid exchange as predictors of second cancers in Hodgkin's disease. Leukemia and Lymphoma 1998;289:561566.

31. Srivastava S, Zou ZQ, Pirollo K, Blattner W, Chang EH. Germline transmission of a mutated p53 gene in a cancer-prone family with Li-Fraumeni syndrome. Nature 1990;348:747749.

32. Lynch HT, Smyrk T. An update on Lynch syndrome. Curr Opin Oncol 1998;10:349-356.

33. Knudson AG Jr. The ninth Gordon Hamilton-Fairley memorial lecture. Hereditary cancers: clues to mechanisms of carcinogenesis. Br J Cancer 1989;59:661-666.

34. Atwater SW, Powers BE, Park RD, Straw RC, Ogilvie GK, Withrow SJ. Thymoma in dogs: 23 cases. J Am Vet Assoc 1994;205:1007-1013.

35. Knowles DM 2nd. Thymoma and chronic myelogenous leukemia: a case report. Cancer 1976;38:1414-1419.

36. Skinnider LF. Alexander S. Horsman D. Concurrent thymoma and lymphoma: a report of two cases. Hum Pathol 1982;13:163-166.

37. Bulkley GB, Bass KN, Stephenson GR, Diener-West M, George S, Reilly PA, Baker RR, Drachman DB. Extended cervicomediastinal thymectomy in the integrated management of myasthenia gravis. Ann Surg 1997;226:324-334.

38. Masaoka A, Yamakawa Y, Niwa H, Fukai I, Saito Y, Tokudome S, Nakahara K, Fujii Y. Thymectomy and Malignancy. Eur J Cardiothoracic Surg 1994;8:251-253.

39. Freidman HD, Inman DA, Hutchinson RE, Poiesz BJ. Concurrent invasive thymoma and T-cell lymphoblastic leukemia and lymphoma. A case report with necropsy findings and literature review of thymoma and associated hematologic neoplasm. Am J Clin Pathol 194;101:432-437.

40. Souadjian JV, Silverstein MN, Titus JL. Morphologic studies of the thymus in human neoplasia. Cancer 1969; 23:619-625.

41. Barton AD. T-cell lymphocytosis associated with lymphocyterich thymoma. Cancer 1997;80:1409-1417. 
42. Gopal AK, Schuetze SM, Maloney DG, Weiden PL. Large cell non-Hodgkin's lymphoma and Hodgkin's disease arising synchronously in a patient with chronic lymphocytic leukemia: importance of immunocytochemistry. Blood. 1999;94:2537.

43. Takaue Y, Sullivan MP, Ramirez I, Cleary KR, van Eys J. Second malignant neoplasm in treated Hodgkin's disease. Report of a patient and scope of the problem. Am J Dis Child 1986;140:49-51.

44. Goh SG, Lau LC, Sivaswaren C, Chuah KL, Tan PH, Lai D. Pseudodicentric $(16 ; 12)(\mathrm{q} 11 ; \mathrm{p} 11.2)$ in a type $\mathrm{AB}$ (mixed) thymoma. Cancer Genet Cytogenet 2001;131:42-47.

45. Zettl A, Strobel P, Wagner K, Katzenberger T, Ott G, Rosenwald A, Peters K, Krein A, Semik M, MullerHermelink HK, Marx A. Recurrent genetic aberrations in thymoma and thymic carcinoma. Am J Pathol 2000;157:257266. 